Revue d'histoire de l'Amérique française

REVUE D.HISTOIRE DE L'AMÉRIQUE FRANÇAISE

\title{
Les avatars de la radio publique d'expression française au Canada 1932-1939
}

\section{Alain Canuel}

Volume 51, numéro 3, hiver 1998

URI : https://id.erudit.org/iderudit/005348ar

DOI : https://doi.org/10.7202/005348ar

Aller au sommaire du numéro

\section{Éditeur(s)}

Institut d'histoire de l'Amérique française

\section{ISSN}

0035-2357 (imprimé)

1492-1383 (numérique)

Découvrir la revue

\section{Citer cet article}

Canuel, A. (1998). Les avatars de la radio publique d'expression française au Canada 1932-1939. Revue d'histoire de l'Amérique française, 51(3), 327-356. https://doi.org/10.7202/005348ar
Résumé de l'article

La radio publique d'expression française au Canada connaît des difficultés au début des années 1930. La question du français sur les ondes nationales devient rapidement un brandon de discorde et la Commission canadienne de la radiodiffusion (CCR), qui a la responsabilité de réglementer et de contrôler l'ensemble de la radiodiffusion au Canada, ne peut véritablement s'imposer face à cette situation conflictuelle. Le présent article retrace les faits marquants qui ont caractérisé l'évolution de la radio d'expression française au Canada en faisant état des pressions exercées à l'endroit de la CCR quant à l'utilisation du français sur les ondes nationales, des mesures administratives jugées discriminatoires à l'endroit de certains gestionnaires francophones et des dissensions parmi les membres de la commission. Il soulève également les difficultés rencontrées lors de la mise sur pied du réseau français de Radio-Canada avant la Deuxième Guerre mondiale.
Tous droits réservés @ Institut d'histoire de l'Amérique française, 1998
Ce document est protégé par la loi sur le droit d'auteur. L'utilisation des services d'Érudit (y compris la reproduction) est assujettie à sa politique d'utilisation que vous pouvez consulter en ligne.

https://apropos.erudit.org/fr/usagers/politique-dutilisation/ 


\title{
LES AVATARS DE LA RADIO PUBLIQUE D'EXPRESSION FRANÇAISE AU CANADA 1932-1939
}

\author{
ALAIN CANUEL \\ Conseil de recherches en sciences naturelles et \\ en génie du Canada
}

\section{RÉSUMÉ}

La radio publique d'expression française au Canada connaît des difficultés au début des années 1930. La question du français sur les ondes nationales devient rapidement un brandon de discorde et la Commission canadienne de la radiodiffusion (CCR), qui a la responsabilité de réglementer et de contrôler l'ensemble de la radiodiffusion au Canada, ne peut véritablement s'imposer face à cette situation conflictuelle. Le présent article retrace les faits marquants qui ont caractérisé l'évolution de la radio d'expression française au Canada en faisant état des pressions exercées à l'endroit de la CCR quant à l'utilisation du français sur les ondes nationales, des mesures administratives jugées discriminatoires à l'endroit de certains gestionnaires francophones et des dissensions parmi les membres de la commission. Il soulève également les difficultés rencontrées lors de la mise sur pied du réseau français de Radio-Canada avant la Deuxième Guerre mondiale.

\section{ABSTRACT}

French language public radio in Canada was faced with major problems in the early 1930s. "The French fact» rapidly became a bone of contention and the Canadian Commission on Radiobroadcasting (CCR), responsible for the setting up of rules and for the control of broadcasting in Canada, was unable to impose its authority in regard to this conflictual situation. The present article relates the main facts that characterized the evolution of French language radio in Canada in focusing on the pressures put on the CCR with respect to the use of the French language on the national network, on administrative measures deemed discriminatory toward certain French administrators, and on dissensions within the Commission. It also discusses some difficulties encountered during the creation of the CBC French network before the Second World War.

\section{INTRODUCTION}

Cet article vise à cerner l'évolution de la radio publique d'expression française au Canada depuis la formation de la Commission cana- 
dienne de la radiodiffusion (CCR) jusqu'au début de la Seconde Guerre mondiale, soit de 1932 à 1939. Notre étude s'inscrit dans la foulée des propos de Frank W. Peers, E. Austin Weir, David Ellis et Margaret Prang qui ont soulevé quelques voiles pour faire état des forces vives, des tensions et des pressions multiples de certains individus, groupes ou organismes à l'endroit des membres de la CCR afin, notamment, de contrecarrer le développement de la radio d'expression française à l'échelle du pays et de confiner cette dernière à l'intérieur des frontières du Québec.

Les interprétations des auteurs susmentionnés ne satisfont que partiellement à notre analyse puisqu'elles s'appuient, dans une large mesure, sur les facteurs politico-économiques qui influencent les décisions et les actions de la CCR et qu'elles considèrent le « fait français » comme un des nombreux avatars de la radio. Dans son ouvrage intitulé The Politics of Canadian Broadcasting, Frank Peers ne consacre que quelques paragraphes à la question du français sur les ondes ${ }^{1}$. À l'instar du président de la CCR, Hector Charlesworth, il dénonce les opposants anglophones qui fustigent le fait français sur les ondes et il rapporte quelques réactions à la Chambre des communes, notamment celles du député de Régina, Franklin W. Turnbull, et la réplique d'un journaliste du Devoir aux propos de Turnbull. Selon Peers, la création du réseau français de Radio-Canada au Québec semble s'inscrire naturellement dans les politiques nationales de la radio alors même que sa création fut, en grande partie, la résultante d'une série d'oppositions et de pressions en provenance du reste du Canada. Dans son article intitulé «The Nationalist Dilemma in Canadian Broadcasting », Peers soutient que le nationalisme et le libre marché ont déterminé pour une bonne part le système de radiodiffusion au Canada et qu'ils représentent deux forces sociales, souvent en conflit. En outre, il reconnaît que les différentes interprétations et hypothèses qui ressortissent aux nationalismes canadien-français et canadien-anglais sont demeurées quasi inexplorées ${ }^{2}$.

Dans son ouvrage intitulé The Struggle for National Broadcasting in Canada, E. Austin Weir prétend que les auditeurs anglophones, en particulier ceux de l'Ontario et de l'Ouest canadien, ne s'opposent pas à la langue française comme telle, mais plutôt à son association au catho-

1. Frank Peers, The Politics of Canadian Broadcasting, 1920-1950 (Toronto, University of Toronto Press, 1969), 128 et ss.

2. Frank Peers, « The Nationalist Dilemma in Canadian Broadcasting », dans Peter Russel, dir., Nationalism in Canada (Toronto, McGraw Hill, 1966), 252-271 et, particulièrement, 252253. 
licisme romain. Pour l'auteur, la diffusion d'émissions en français sur les ondes nationales à un moment où la colère de l'auditoire anglophone est nourrie par un mélange de préjugés, de bigoterie et de peur représente de loin l'incident le plus regrettable qui soit de la radiodiffusion nationale ${ }^{3}$. Cette brève explication qui tient à quelques paragraphes suffit à l'auteur pour cerner le «French fact » et blâmer avec diplomatie la CCR pour son manque d'expérience, en particulier Thomas Maher ${ }^{4}$.

Dans un court chapitre intitulé « Grandeur et décadence de la Commission canadienne de la radiodiffusion », David Ellis fait état du problème du français $[s i c]$ en précisant qu'il représente un des nombreux motifs qui viennent accentuer les critiques envers la CCR. Il considère que le problème du français au réseau national fait partie des « incidents » qui n'auraient pas eu une telle importance si la CCR « avait disposé des ressources et de l'esprit d'initiative indispensables à la construction de la chaîne de stations très puissantes $[\ldots]^{5}$ ».

Margaret Prang, pour sa part, analyse le développement de la radio publique au Canada par rapport à l'attitude de l'entreprise privée qui hésite à prendre des risques étant donné l'étendue du pays et sa faible population et par rapport à la volonté de certains groupes canadiens qui pressent le gouvernement de développer une forme de sécurité nationale face à l'attitude économique et politique des États-Unis ${ }^{6}$. L'auteure ne soulève pas explicitement la question de la radio d'expression française au Canada. S'appuyant principalement sur une opposition socioculturelle et économique entre le Canada et les États-Unis, l'analyse de Prang tend à gommer la dimension française de la radio nationale en

3. E. Austin Weir, The Struggle for National Broadcasting in Canada (Toronto, McClelland \& Stewart Ltd., 1965), 149-151.

4. Et pour cause! Austin Weir, directeur de la radio du Canadien national se joindra à la CCR à titre de directeur des émissions. Cette fonction administrative sera de courte durée. En février 1933, sous les instances de Thomas Maher, Weir est limogé et remplacé par J.-Arthur Dupont de la station CKAC, à Montréal. Pour une meilleure compréhension de ces événements voir les références suivantes: Canada, House of Commons, 1934, Special Committee on the Operations of the Commission under the Radio Broadcasting Act 1932 (as amended), Minutes of Proceedings \& Evidence, 11 (18 mai 1934): 434-458; 13 (31 mai 1934): 537-559 (ci-après nommé ANC, 1934 Proceedings); Archives nationales du Canada, Fonds Bennett, MG26K, 590 (13 mars 1933): 368457-368459 (ci-après nommé ANC).

5. David Ellis, La radiodiffusion canadienne: objectifs et réalités, 1928-1968 (Hull, Centre d'édition du gouvernement du Canada, 1979), 14.

6. Margaret Prang, «The Origins of Public Broadcasting in Canada », Canadian Historical Review, 46,1 (1975): 1-31. Cette approche n'est pas nouvelle. Frank Peers affirme que la commission royale de la radiodiffusion (1928) «n'a pas fait référence au problème d'émissions produites pour le tiers de la population canadienne-française ». Peers, The Politics of..., op. cit., 49. 
l'inscrivant dans un tout indivisible, c'est-à-dire « canadien » plutôt que spécifiquement canadien-anglais et canadien-français.

La radio publique d'expression française est ainsi saisie et interprétée comme une sorte d'appendice du réseau national. Les auteurs qui se sont penchés sur les débuts de la radio publique au Canada n'ont pas, selon nous, accordé suffisamment d'importance au fait français, occultant ainsi une dimension importante de l'histoire de la radio publique au Canada. Notre analyse se propose de scruter certains événements marquants de l'administration quotidienne de la CCR en faisant état des pressions exercées à son endroit quant à l'utilisation du français sur les ondes nationales, des mesures administratives jugées discriminatoires à l'endroit de certains gestionnaires francophones et des dissensions parmi les membres de la commission. Ce regard de l'intérieur nous permettra d'éclairer certaines décisions et choix politiques qui ont conduit à la nationalisation de la radio au Canada, en particulier la création du réseau français de la Société Radio-Canada (SRC). Bien que la création de ce réseau constitue une véritable enclave en territoire national, notre analyse permettra de caractériser la radio publique d'expression française dans ses rapports à l'échelle nationale. Au terme de notre étude, nous verrons que cette évolution aura permis au réseau français de cristalliser ses forces au sein de la SRC et de développer une spécificité propre à la langue et à la culture d'expression française au Canada.

Notre démarche analytique ne constitue pas une sorte d'inventaire d'événements à partir desquels nous pourrions poser des rapports dichotomiques entre les tenants de la radio publique d'expression française et ses opposants. Qui plus est, les différences que nous établissons entre ces deux catégories d'acteurs ne peuvent être définies uniquement sur la base de considérations linguistiques et culturelles. Notre étude s'inscrit dans une perspective plus large du fait qu'elle se dissocie de la pensée de certains historiens, tels Walter B. Emery et Frank H. Underhill, qui prétendent que le biculturalisme et, particulièrement, le provincialisme ont travaillé contre le développement d'institutions nationales fortes, que la particularité d'une petite population occupant une infime portion sur ce continent a imputé au Canada des coûts supplémentaires et que, inévitablement, cela a affaibli le sens d'une véritable unité nationale ${ }^{7}$.

7. Walter B. Emery, «Canadian Broadcasting: Unity and Diversity », dans Walter B. Emery, dir., National and International Systems of Broadcasting: their History, Operation, and Control (Michigan, Michigan University Press, 1969), 44; Frank W. Underhill, « The Price of Being Canadian », dans D. L. B. Hamlin, dir., The Price of Being Canadian, 7th Winter Conference (Toronto, University of Toronto Press, The Canadian Institute of Public Affairs, 1961), 10-12. 


\section{1 - LA COMMISSION CANADIENNE DE LA RADIODIFFUSION, 1932- 1936}

En 1928, le gouvernement canadien met sur pied une commission royale d'enquête, nommée commission Aird, dont le mandat est de « connaître les conditions de la radiodiffusion au Canada et de formuler des suggestions relativement à l'administration, à la direction et à la surveillance et aux besoins financiers de ce service ${ }^{8} »$. Les recommandations présentées par la commission Aird indiquent un besoin pressant de créer une chaîne de radiodiffusion entièrement canadienne qui serait supervisée par un organisme fédéral indépendant. Malgré l'effort méritoire de ce rapport, la crise de 1929 aura vite fait de polariser l'attention des politiciens vers des préoccupations plus pressantes et l'institution d'un comité parlementaire chargé d'étudier la situation n'aboutira que trois ans plus tard. Le 16 février 1932, le Premier ministre, Richard Bedford Bennett, déclare à la Chambre des communes:

On doit convenir que le régime actuel de radiodiffusion ne donne pas satisfaction. Les Canadiens ont droit à une radiodiffusion de source canadienne, égale à tous égards à celle de tout autre pays. On ne pourra établir un tel régime qu'après l'enquête la plus approfondie, et en conformité d'un programme dont l'exécution demandera plusieurs années. Il est bien clair qu'il sortira des avantages immenses d'un régime de radiodiffusion convenable, réglementé et exploité par des Canadiens. Employée comme il convient, la radio peut devenir un instrument très efficace au service du progrès national et prendre une valeur éducative qu'il est difficile d'évaluer'.

Des deux côtés de la Chambre, l'on reconnaît enfin l'urgente nécessité de contrôler et de réglementer la radiodiffusion au Canada. Pour débattre de cette question complexe, le gouvernement Bennett présente, le 18 mai 1932, un bill visant à établir la Commission canadienne de la radiodiffusion. En vertu de son mandat, la CCR s'engage à examiner les recommandations du rapport Aird, à soumettre un plan technique complet et à proposer le moyen le plus satisfaisant pour sa réalisation. La double tâche de la CCR, qui consiste à réglementer et à contrôler l'ensemble de la radiodiffusion au Canada, puis à exploiter un réseau national, ne peut être accomplie sans le moindre manquement. Cette restructuration de la radio qui incombe aux commissaires soulève

8. Canada, Ministère de la Marine, Rapport de la Commission royale de la radiodiffusion (Ottawa, A. F. Acland, 1928-1929), 5.

9. Canada, Chambre des communes, Débats, 16 février 1932, 1: 231-232. 
d'importantes difficultés techniques et financières souventes fois liées à leur pouvoir réglementaire. Sur le plan technique, il faut mettre en œuvre des changements de fréquences tout en demeurant dans les limites d'ondes permises par l'Accord international de Madrid (1932); surmonter le handicap géographique auquel est confrontée la radio pour couvrir d'est en ouest le pays; prévoir un décalage horaire pour les émissions diffusées à l'échelle nationale; offrir un service aux deux principales communautés culturelles du pays; et régler le contentieux affectant particulièrement les stations limitrophes. Sur le plan financier, il importe de fixer et de répartir les sommes nécessaires à l'administration et aux dépenses de la commission et surtout d'administrer les crédits budgétaires nettement insuffisants pour la construction de nouvelles stations, l'achat ou la location de postes privés, les contrats de liaison par fil avec les compagnies privées, de même que les dépenses affectées aux émissions radiophoniques (droits d'auteurs, cachets des artistes, orchestre, etc.). Pour faire face à ses obligations, la commission doit compter uniquement sur les crédits alloués par le Parlement et ne peut, en principe, excéder son budget sans l'assentiment du gouvernement. Elle est, entre autres, assujettie à la loi de la fonction publique pour l'engagement de techniciens spécialisés, d'artistes ou annonceurs, ce qui gêne considérablement ses activités ${ }^{10}$.

Ainsi, il n'est pas étonnant de constater que la CCR ait peine à soutenir sa réputation. À l'automne de 1933, R. W. Ashcroft, ancien viceprésident de l'Association canadienne des radiodiffuseurs (ARC), affirme que: «[...] l'exercice de son autorité [CCR] semble n'avoir produit que de piètres résultats. La commission n'a obtenu aucun appui et n'a pas su se mériter un geste de sympathie à tous égards, et ceux qui avaient louangé sa création sont désormais opposés définitivement à sa continuation ${ }^{11}$. »Cette affirmation laisse poindre de graves difficultés quant aux conditions dans lesquelles la commission devra exercer son autorité jusqu'à la fin de son mandat.

Dès la création de la CCR, les membres de la commission, que sont le président, Hector Charlesworth (rédacteur en chef du Saturday Night de Toronto), le vice-président, Thomas Maher (ingénieur forestier), le commissaire William Arthur Steel (ingénieur-radio), détaché par le ministre de la Défense nationale, et le secrétaire, le lieutenant-colonel René-P. Landry, participent activement à la diffusion et à la réalisation

10. ANC, Fonds Murray, MG 30 E186, 1, « CBC appointments », 1-2.

11. ANC, Fonds A. W. Steel, MG 30 A42, 28, Dossier 158, « A plan for improved broadcasting in Canada », 1. 
d'émissions nationales. À la fin de 1932, la CCR acquiert les studios du Canadien national et peut enfin commencer, un mois plus tard, à recruter son personnel et à mettre en service ses stations de Vancouver, Toronto, Montréal, Ottawa et Chicoutimi. Dès la première semaine de mai 1933, une programmation quotidienne fait son apparition à l'échelle nationale, ce qui n'empêche pas la commission d'être sévèrement critiquée pour son zèle face à l'utilisation du réseau pour des émissions en français et pour sa piètre performance dans la production d'émissions nationales. Les commissaires qui dirigent à la fois l'exploitation et la gestion du réseau national ne peuvent manifestement suffire à la tâche. Ils seront en butte à des récriminations sans nombre qu'ils ne pourront contenir parce que trop souvent liées à leur personnalité, à la structure ou au mandat même de la CCR.

Quelques jours seulement après la mise sur pied de la programmation nationale, certains groupes anglophones manifestent déjà leur profond désaccord quant à la diffusion d'émissions en français sur les ondes nationales. Dans sa livraison du 20 mai 1933, le journal Evening Telegram titre: «Les citoyens protestent contre la langue française ». Les signataires de l'article qui représentent les Orangistes dénoncent la pratique grandissante d'émissions et de commentaires en français qu'autorise la commission. De vives réactions ne tardent pas à se multiplier dans les provinces anglophones et, devant le tollé, la CCR se voit obligée de justifier sa position face à l'utilisation du français sur les ondes $^{12}$.

L'une des premières réponses aux attaques soutenues des Orangistes paraît dans le journal Mail \& Empire (24 juin 1933). Les émissions nationales, affirme la commission, totalisent cinq heures et demie en anglais et deux heures en français. Du dimanche au samedi, «l'heure nationale »a été répartie selon le ratio établi. Le dimanche soir, l'émission provient alternativement de Montréal et de Toronto. Lorsque l'émission provient de Montréal, soutient la commission, nous présentons une heure d'opérette en français et une demi-heure de contenu divers en anglais. Lorsqu'elle provient de Toronto, nous diffusons une

12. Voir les références suivantes: «Three Hours a Day of Dominion Radio », Mail \& Empire, 25 juillet 1933; «New Deal Seen in Federal Radio for Near Future », Financial Post, 9 septembre 1933, 8-9; E. A. Weir, The Politics of..., op. cit., et, particulièrement, le chapitre 12 «The Rumpus Over Programming in French », 149 et ss.; ANC, Fonds Bennett, MG26K, 588: (26 juillet 1933), 366603. 
heure complète en anglais ${ }^{13}$. Cette justification de la commission ne suffit pas à calmer les esprits, d'autant plus que la CCR fait connaître quelques jours plus tard sa programmation spéciale pour souligner le $66^{\mathrm{e}}$ anniversaire de la Confédération canadienne, laquelle débute par le $\hat{O}$ Canada en français ${ }^{14}$. Les attaques de groupes opposés au français sur les ondes se multiplient et elles sont de plus en plus dirigées contre Thomas Maher, responsable de la programmation nationale, que l'on identifie de plus en plus ouvertement comme le promoteur des intérêts des francophones au Canada. L'utilisation du français sur les ondes ne tarde pas à ramener à fleur de mémoire des propos choquants à l'égard du peuple canadien-français, alors que les opposants exercent des pressions dans le but déclaré de promouvoir « les principes britanniques et la langue anglaise ${ }^{15} »$.

À l'été de 1933, Thomas Maher annonce à la presse que le projet d'expansion du réseau national, en particulier dans certaines régions isolées du Québec, permettra, par le biais des arts et de l'éducation, de créer un Canada uni et de développer une meilleure compréhension entre les provinces canadiennes de l'Est et de l'Ouest. Par la même occasion, il déclare que le territoire du Québec sera bientôt desservi par des stations qui diffuseront majoritairement en français. Cette mesure, affirme-t-il, permettra de rejoindre les auditeurs dans un très grand rayon ${ }^{16}$. Cette déclaration, qui s'appuie sur le principe de la double appartenance linguistique et culturelle du Canada de même que sur l'équité d'un service radiophonique dans les diverses régions du pays, permet de justifier l'apport du français sur les ondes et de promouvoir une meilleure compréhension entre les deux peuples fondateurs. «Les citoyens d'origine française et anglo-saxonne au Canada sont des Canadiens à part entière, soutient Maher, et ceux qui proclament qu'il y a beaucoup trop de français dans la programmation nationale ne peuvent réellement prouver la prédominance de cette langue, compte tenu de la

\footnotetext{
13. «Radio Board Answers Orange Order Protest», Mail \& Empire, 24 juin 1933. Nous analyserons plus en détail la répartition du temps d'antenne des émissions en français et en anglais dans la troisième partie de notre analyse.

14. «Commission Offers 'O Canada' in French as Our National Holiday Program Opener », Evening Telegram, 29 juin 1933.

15. «Oppose Radio French Policy - York L.O.L. 375 Has Many Veteran Orange Leaders », Evening Telegram, 12 juillet 1933; «Commission to Give What People Want. French Canadian Member Wants to Please Most Ardent Orangemen », Toronto Star, 14 juillet 1933; « Three Hours a Day of Dominion Radio », Mail \& Empire, 25 juillet 1933; «Will Continue French on Radio Programs », Evening Telegram, 25 juillet 1933.

16. «Quebec Radio Plans Cover Wide Field», Globe \& Mail, 1er juillet 1933. À noter que le plan annoncé par Maher ne se concrétisera pas au cours de son mandat à la commission.
} 
proportion [que nous avons] établie ${ }^{17}$. $\gg$ Les arguments de Maher ne réussissent toutefois pas à convaincre l'opinion publique anglophone du bien-fondé d'émissions en français. À la suite des attaques répétées de l'Ontario et des provinces de l'Ouest, la controverse du français sur les ondes atteint son point culminant au printemps de 1934.

L'un des faits marquants de cette période houleuse est, sans contredit, le témoignage du député de Régina, Franklin W. Turnbull, devant le comité spécial de 1934 chargé de s'enquérir des opérations de la CCR. Au cours de son apparition devant le comité, Turnbull fait état des résultats d'un sondage commandé par le journal Regina Star: $40 \%$ des répondants, déclare-t-il, ont comme principale objection l'utilisation du français lors de la présentation d'émissions tandis que $87 \%$ s'opposent au français sous cette forme ou sous une autre. Selon l'opinion de la population de la Saskatchewan, la langue française ne doit pas être considérée comme une langue officielle pour tout le Canada et son utilisation doit être limitée aux conditions stipulées dans l'Acte de l'Amérique du Nord britannique. Avec une habileté politique consommée, Turnbull fait part à la Commission d'une résolution prise par The Sons of England of Prince Albert à l'effet que «l'utilisation du français en dehors du Québec se veut un effort concerté des gens d'origine française pour faire du Canada un pays bilingue ${ }^{18}{ }$. Le député de Régina orchestre ainsi une campagne de dénigrement du français en utilisant l'arène politique pour rallier l'opinion publique anglophone.

Certains protagonistes qui, jusqu'ici, avaient fait preuve de mesure et de retenue sur la question du français sur les ondes furent irrités par les propos vexateurs du député Turnbull. Paul Auger, journaliste au Devoir, écrit: «Trente secondes de français dans un programme de radio d'État leur met l'écume à la bouche, la rage au cour et, parfois, la torche incendiaire au poing ${ }^{19}$. » Il n'hésite pas à dénoncer Turnbull comme l'un des principaux leaders du Ku Klux Klan, groupe qu'il compare volontiers à des fossiles microcéphaliques. Hector Charlesworth qualifie cette organisation américaine de bâtarde en affirmant que trois membres se sont infiltrés à la Chambre des communes et que l'un affiche ouvertement son appartenance (en l'occurrence, F. W. Turnbull), tandis que les deux autres s'en abstiennent. Il soutient que ces personnes ont provoqué un mouvement d'agitation au sein des Orangistes en pro-

17. « Three Hours a Day of Dominion Radio », Mail \& Empire, 25 juillet 1933.

18. ANC, 1934 Proceedings, op. cit., 494-505 et, particulièrement, 499.

19. «L'actualité, M. Turnbull », Le Devoir, 31 mai 1934. 
clamant que l'utilisation du français sur les ondes venait de faire une brèche à l'Acte de l'Amérique du Nord britannique ${ }^{20}$.

Les attaques répétées de Turnbull pour libérer les ondes nationales $\mathrm{du}$ français, en particulier dans l'Ouest canadien, ne laissent pas indifférent le Premier ministre Bennett. La liberté d'action totale que Bennett garantissait au président de la CCR avant même que ce dernier n'entre en fonction (« en vous offrant cette position, Charlesworth, je vous offre un chèque en blanc où vous serez libre d'agir comme bon vous semble $\left.{ }^{21} \gg\right)$ est remise en question. En 1933, la venue de Gladstone Murray de la British Broadcasting Corporation, à titre de conseiller spécial du Premier ministre canadien, est révélatrice des difficultés que rencontre la CCR et des inquiétudes politiques que cette commission soulève. À cet effet, Murray présente trois rapports au Premier ministre concernant l'organisation et le mandat de la commission et formule des recommandations sur les pouvoirs administratifs de la commission ${ }^{22}$. Quelques semaines plus tard, Thomas Maher fait parvenir une note de service à Bennett dans laquelle il soulève, entre autres, la question du français sur les ondes et l'absence de stratégie et de publicité pour contrecarrer les attaques dirigées contre les émissions en français, en concluant que cette faiblesse pourrait conduire à la disparition de la CCR. Maher soutient, en outre, que la diffusion d'émissions en français représente trois heures et demie comparativement à quarante-cinq heures et demie pour celles en anglais, sur un total de 49 heures/semaine ${ }^{23}$. La crise du français sur les ondes qui s'ajoute aux problèmes de la commission indispose Bennett qui manifeste à Maher son insatisfaction: « $\grave{A}$ mon avis, écrit le Premier ministre, aucune raison ne peut justifier la situation actuelle dont vous êtes responsable et ne peut constituer une excuse valable face à ce qui vient de se passer ${ }^{24}$. »

Les forces politiques, l'influence des journaux et même les pétitions qui appuient le fait français sur les ondes nationales ne peuvent véritablement contrebalancer l'opinion publique anglophone et ses agitateurs.

20. Hector Charlesworth, I'm Telling You (Toronto, MacMillan, 1937), 99; voir également, «"From Ladies" Orange Benevolent Association Club to R. B. Bennett, W. L. Mackenzie King, Hector Charlesworth », ANC, Fonds Bennett, op. cit., 366342, 22 octobre 1933.

21. Charlesworth, I'm Telling You, op. cit., 34.

22. ANC, RG14, D-1, Appendices, 680, « National Radio in Canada », Report by Major Gladstone Murray, Director of Public Relations, British Broadcasting Corporation, July 25, 1933, voir particulièrement, « The Organization Problem », 6 et ss.; Canada, House of Commons, Debates, 11 mai 1933, 4861, 4872 (ci-après nommé Debates).

23. ANC, Fonds Bennett, 590 (14 septembre 1934): 365254-365257.

24. Ibid., 16 septembre $1934,365260$. 
Qui plus est, d'autres groupes alimentent la controverse mais, cette fois, pour des raisons différentes. Par exemple, le Evening Telegram, qui par sa position radicale nourrit la controverse du français, sera la cible du président de la CCR qui prétend que ce journal est « un organe de R. W. Ashcroft, président de l'Association canadienne des radiodiffuseurs et directeur de la station CKGW de Toronto ${ }^{25} »$. N'y a-t-il pas lieu de penser que la bataille livrée à la commission par les puissants propriétaires de stations privées, notamment au chapitre de la diffusion d'émissions non commanditées dont le tarif se situe bien au-dessous de celui de leur minimum commercial, serve de toile de fond à la controverse du français pour miner la crédibilité et du même coup neutraliser les pouvoirs statutaires de la CCR? René-P. Landry, secrétaire de la commission, soutient en effet que «les adversaires de la nationalisation sont pour quelque peu responsables de cet état de chose; n'atteindraient-ils pas leur but s'ils réussissaient à faire abolir la Commission en soulevant les préjugés de race ${ }^{26}$ ? »

Pour les propriétaires de stations privées, les enjeux financiers sont énormes et ils doivent livrer bataille tous azimuts. La politique de la CCR qui vise à promouvoir le français sur les ondes nationales devient particulièrement gênante pour certains radiodiffuseurs privés. Au début de 1932, quatre stations privées canadiennes (CKAC, CJAD [Montréal] et CFRB, CFCA [Toronto]) vont s'affilier au réseau américain CBS pour combler jusqu'à la moitié de leur programmation quotidienne. Les stations de Montréal, et particulièrement CKAC qui rejoint par sa puissance émettrice près d'un million d'auditeurs francophones au Québec, dans l'est de l'Ontario, dans une partie des Maritimes et même dans les États de la Nouvelle-Angleterre, ne répondent manifestement pas aux attentes de la CCR ni à celles du comité spécial de la radiodiffusion de 1932.

25. « Radio Chairman Says Telegram Ashcroft Organ », Evening Telegram, 20 avril 1934.

26. ANC, Fonds René-P. Landry, MG 30, E326, vol. I, « Mémoire de Landry adressé à Maher », 25 septembre 1933. Depuis le début des années 1930, la publicité sur les ondes est remise en question, entre autres, par la Ligue canadienne de la radio et par la commission spéciale sur la radiodiffusion de 1932. Cette situation provoque d'énormes tensions entre les partisans de la radio publique qui rejettent toute forme de publicité et les radiodiffuseurs privés, majoritairement propriétaires de grands journaux. Sur cette question, voir les principales références suivantes: ANC, Fonds Thomas James Allard, MG 30, D304, vol. 6, Dossier 16, document C-18, « The Canadian Radio League »; vol. 5, Dossier 9, «Extract from a Brief Respecting Radio Broadcasting Submitted to the Royal Commission on Radio Broadcasting in Toronto, May 17, 1929 by Canadian Manufacturing Association »; ANC, Canadian Association of Broadcasters, MG 28, I 363, vol. 3, Dossier C-1-1, « Discussion Concerning the Canadian Radio Commission »; vol. 4, Dossier C-78, « Lettre de T.-J. Allard à Lucille Birchaud », 16 décembre 1957. 
Le commentaire qu'exprime Grant Dexter, correspondant du journal Winnipeg Free Press à Ottawa, en septembre 1933, est symptomatique des événements futurs: «Le nombre d'émissions de langue anglaise [sur les ondes nationales] a rapidement augmenté tandis que le nombre d'émissions de langue française a diminué27. »Quelques mois plus tard, Ernest Bushnell, directeur de la programmation pour la région de l'Ouest canadien soutient que 75 à $80 \%$ de toutes les émissions diffusées au Québec sont entièrement en anglais ${ }^{28}$. La pauvreté du français sur les ondes nationales, la piètre qualité des services offerts en français au Québec de même que l'opinion publique anglophone acquise à l'idée que le français ne devrait pas dépasser les frontières du Québec - Paul Anger du Devoir illustre ce propos en faisant référence au cliché de la « réserve indienne »- ne permettent pas à la radio d'expression française de se développer, comme le souhaitait Maher, sur la base d'une meilleure compréhension des deux peuples fondateurs et encore moins sur le principe d'équité d'un service radiophonique dans les diverses régions du pays.

La nationalisation de la radio ne devient une unité parfaite qu'au prix du rejet, sinon de l'antipathie d'une partie de la population canadienne vis-à-vis de la langue et de la culture d'expression française. À cet égard, le discours de Turnbull demeure significatif puisqu'il permet de faire ressortir deux principes que nous avons déjà soulignés et que nous retrouvons avec plus ou moins d'ardeur dans les déclarations, témoignages et autres formes d'expression de l'opinion publique anglophone et des ténors de la politique, du journalisme et de l'entreprise privée qui manifestent leur appui à la lutte contre le français. Le premier principe repose sur la certitude que la langue française n'est pas une langue officielle et, par conséquent, que l'on doit s'en tenir à l'application

27. «A Most Unfortunate History », Winnipeg Free Press, 7 et 9 septembre 1933 (première et deuxième parties, section éditorial). Le journaliste fait état de deux heures/semaine d'émissions en français sur les ondes nationales.

28. ANC, 1934 Proceedings, op. cit., 20 avril 1934, 283. Voir également Le Devoir, 11 décembre 1934, dans lequel le journaliste affirme que CKAC diffuse $80 \%$ du temps d'antenne en anglais et demande que la radio nationale vienne à la rescousse des auditeurs francophones. À noter que la CCR retiendra, dès 1933, les services de J.-A. Dupont, directeur de CKAC, et E. L. Bushnell ( $\mathrm{CKCN}$, Toronto) qui avaient été, au cours de la dernière décennie, des ardents défenseurs de la radio privée auprès de la CCR, du comité spécial de la radiodiffusion (1932) et de la Ligue canadienne de la radio. Sur le rôle qu'ont joué Dupont et Bushnell au sein de l'Association des radiodiffuseurs canadiens, voir les ouvrages suivants: Thomas J. Allard, L'histoire de l'ARC, 1926-1976: radio et télédiffusion privée au Canada (Ottawa, 1976), 13, publiée par l'Association canadienne des radiodiffuseurs à l'occasion de son 50e anniversaire; Thomas J. Allard, Straight Up, Private Broadcasting in Canada, 1918-1958 (Ottawa, Canadian Communications Foundation, 1979). 
de l'Acte de l'Amérique du Nord britannique. Le second s'applique à démontrer que le français ne doit pas être entendu en dehors des frontières du Québec. De ces deux principes découlent les corollaires suivants: d'une part, la CCR n'est pas tenue d'observer la règle de présentation des émissions dans les deux langues comme au Québec, puisque les francophones hors Québec comprennent l'anglais et qu'il est inutile d'utiliser le français pour la majorité anglophone; d'autre part, si les auditeurs anglophones syntonisent les stations américaines, cela est imputable aux émissions en français qui envahissent les ondes nationales. Selon le tableau 1, le temps d'antenne consacré aux émissions en français représente, à l'échelle nationale, $14 \%$ de l'ensemble des émissions hebdomadaires.

\section{TABLEAU 1}

\section{RÉPARTITION DES ÉMISSIONS AU RÉSEAU RADIOPHONIQUE NATIONAL}

Semaine du 18 au 24 février 1934

- Nombre d'émissions: 115

- 8 émissions en français (principalement, mais non entièrement en français) aux réseaux national et régional

- 13 émissions en français (réseau limité aux territoires d'expression française)

- 94 émissions en anglais (un rappel à caractère musical indique que les émissions peuvent être soit en anglais soit en français)

TEMPS D'ANTENNE

- émissions en anglais et en français total: 54 heures et 10 minutes

- 8 émissions en français total: 4 heures et 10 minutes

- 13 émissions en français total: 4 heures et 30 minutes

- 94 émissions en anglais total: 45 heures et 29 minutes

Source: ANC, Fonds Bennett, MG26K, vol. 590: 364390-92.

Devant l'offensive des opposants au français sur les ondes nationales, une solution politique s'impose. A priori, celle qui semble la plus rentable pour Bennett, s'il ne veut pas s'aliéner l'électorat québécois et s'il désire satisfaire l'opinion publique anglophone, est d'offrir aux Qué- 
bécois une programmation en français séparée du réseau ${ }^{29}$. Cette orientation ne se fera pas sans heurts. Les différents acteurs sociaux qui ont proclamé que la radio était un instrument d'unité nationale, un moyen de contrecarrer la présence américaine sur les ondes canadiennes et qui ont appuyé ce principe depuis la commission Aird font face désormais à une distorsion de plus en plus grande entre la poursuite d'un idéal politique et social et l'établissement d'un réseau radiophonique national. Dans le tumulte des passions, la vision de l'unité nationale qui se dégage de la radio s'appuie sur diverses interprétations selon les intérêts ou les convictions qu'on y défend. Pour tenter de rétablir l'équilibre entre les tenants du français sur les ondes nationales et ses opposants, la CCR oriente depuis 1933 ses activités vers le développement d'un réseau bicéphale, espérant ainsi concilier les intérêts des uns qui ont partagé la vision d'une unité nationale basée sur la compréhension mutuelle des deux peuples fondateurs et des autres qui voient en la radio un instrument de domination de la race française. La démission de Thomas Maher qui a provoqué une rupture au sein de la commission en 1934 et qui coïncide avec le retrait des émissions en français sur les ondes nationales ne laisse pas les protagonistes indifférents ${ }^{30}$. Officiellement, Maher quitte la CCR pour des raisons de santé, mais les pressions exercées à son endroit à titre de responsable de la programmation nationale et son profond désaccord avec Charlesworth et Steel sur l'orientation de la radio auront certainement précipité son départ ${ }^{31}$.

Bien que la démission de Maher ait favorisé un repli du français, son action au sein de la commission marque un jalon important dans l'histoire de la radio au Canada. Un journaliste de Chicoutimi décrit en ces termes le rôle de Maher:

Dans le travail d'organisation accompli par la CCR, M. Maher, qui fut vice-président a certainement une très large part de mérite. S'il y a du français dans l'air qui s'irradie en dehors de Québec, si les programmes de la Commission portent par tout le Canada

29. Dans une lettre adressée au ministre de la Marine, Alfred Duranleau, le député Turnbull écrit: « le gouvernement est en train de perdre des centaines de votes à cause de l'insistance des Canadiens français sur le bilinguisme, notamment dans le domaine de la radio [...] Quelques-uns vont même jusqu'à dire qu'il s'agit-là de la plus grande difficulté que connaîtra le gouvernement Bennett lors des prochaines élections. » ANC, Fonds Bennett, op. cit., 13 avril 1934, 365695.

30. Au dire du correspondant du journal Financial Post à Ottawa, les émissions en français au réseau national auraient été retirées à la suite de l'intervention du Premier ministre, voir « New Deal Seen in Federal Radio for Near Future », Financial Post, 9 septembre 1933.

31. Peers, The politics of..., op. cit., 149-150; «Vice-Chairman on Radio Board Seeks Release », Mail \& Empire, 7 juillet 1934; « Maher Proposed to Quit in April », Mail \& Empire, 10 juillet 1934. 
l'étiquette bilingue, cela est bien dû à Maher et pas à un autre. Le mérite n'est pas si mince: quel tapage et quel temps il a fallu pour obtenir un mot de français sur les timbres-postes ${ }^{32}$ !

«L'étiquette bilingue » est certes l'expression idoine pour qualifier la présentation d'émissions au réseau national puisque le temps d'antenne correspond tout au plus à 30 secondes de français sur les ondes. Bien sûr, il y a quelques émissions françaises en provenance de Montréal. Cela s'entend et l'on ne saurait passer sous silence cette distinction entre la présentation et le contenu d'émissions. Toutefois, le témoignage de Turnbull devant le comité spécial de 1934 fait ressortir un aspect important de la question: les 30 secondes témoignent d'une épreuve de force puisque toute intrusion du français à l'échelle nationale est vue comme une violation des droits de la majorité anglophone. Ainsi, la comparaison avec le timbre-poste prend toute sa signification. La radio, le train, les écoles et même certains ministères font l'objet de vives critiques: «[...] nous devons faire échec au bilinguisme français dans les écoles ainsi que dans les nombreux ministères du gouvernement et surveiller de près l'invasion du français au nord de l'Ontario ${ }^{33}$. » Un hebdomadaire de Stouffville, Ontario, publie: « [...] nous préférons des émissions américaines, aussi tarées soient-elles, plutôt que la diffusion [d'émissions] de la CCR dans les deux langues, laquelle semble résolument donner au Canada un caractère bilingue ${ }^{34}$. »

La question du français à la radio s'inscrit dans un débat plus large où les institutions, les organismes nationaux et les ministères fédéraux doivent s'ajuster à la réalité linguistique canadienne. À cet égard, le gouvernement Bennett doit intervenir, puisqu'il est en butte à des récriminations sans nombre autant chez les francophones que les anglophones. Le débat qui a pris place en février 1934 sur l'émission de la monnaie, des notes et des obligations en langue anglaise dans les banques canadiennes servira de toile de fond à la controverse de la radio. Le Premier ministre Bennett a, en effet, constitué un comité spécial chargé d'étudier la question et de lui soumettre ses recommandations. Bien que l'action politique de Bennett ait permis de dénouer la crise du bilinguisme à propos de l'émission de la monnaie, nous devons cepen-

32. «Année radiophonique - Commission de la Radio - M. Maher - CRCS Chicoutimi », Progrès du Saguenay, 11 octobre 1934; voir également: ANC, Fonds Bennett, MG26K, vol. 590, 365279; « Maher défenseur de la langue française », lettre d'Émile Poissant à Thomas Maher, 18 septembre 1933.

33. "Defend Public Schools and Insist on English Language Only, Says Grand Master », Evening Telegram, 31 mars 1934.

34. Peers, The Politics of..., op. cit., 249. 
dant préciser que les députés de la Chambre des communes rejetèrent la recommandation originale qui favorisait l'utilisation simultanée des deux langues dans l'impression des billets. À la suite d'un amendement proposé, la Chambre vota en faveur de l'émission de « billets libellés en anglais et en français selon [la demande] ${ }^{35}{ }$. Cette solution politique au problème du bilinguisme, caractérisée par la duplication, se reflétera désormais dans les différentes sphères d'activités des services nationaux. À cet égard, la radio n'y échappe pas. À preuve, quinze ans plus tard, Paul Legendre fait remarquer que:

La radio se divise en deux zones linguistiques (provinces anglaises et province de Québec) et les influences ne se compénètrent pas. Elles sont à sens unique. En effet, alors que les postes anglais du pays ne diffusent qu'en langue anglaise et font de très rares emprunts à notre radiophonie, la minorité française subit largement les influences anglo-saxonnes ${ }^{36}$.

La CCR n'est pas à l'abri des pressions externes, d'autant plus qu'elle est accablée par des problèmes qui relèvent des affaires courantes de son administration. Il n'est alors pas étonnant de constater que la nouvelle de la démission de Maher, qui s'appuie officiellement sur des raisons de santé, a vite fait d'être interprétée comme une rupture au sein de la CCR. La forte personnalité des membres, les pressions externes et les problèmes d'ordre administratif qui entachent la réputation de la commission conduisent inéluctablement à des différends entre les membres. Les commissaires, qui doivent diriger à la fois l'exploitation et la gestion du réseau national, ne peuvent manifestement suffire à la tâche et se voient parfois contraints d'agir trop rapidement, sans consultation avec les autres membres. L'action de Charlesworth, en mai 1933, est un exemple frappant. En l'absence de Maher, le président émet des directives pour que l'émission en français du dimanche ne soit pas diffusée au-delà de l'Ontario, cédant ainsi aux pressions des députés de

35. Canada, Chambre des communes, Sessions de 1934, Appendice 2, Comité permanent de la banque et du commerce. La Loi 18, intitulée «Loi concernant les banques et le commerce de banque », et la Loi 19, intitulée « Loi constituant en corporation la Banque du Canada », Procèsverbaux, témoignages et recommandations, cvii-cviii; voir également Canada, House of Commons, Journals, Sessionnal Papers, Session 23, LXXII (1934): 126.

36. Paul Legendre, La radio, puissance sociale (Ottawa, Institut littéraire du Québec, 1951), 205. En réalité, cette situation ne changera guère au cours des décennies suivantes. A. W. Johnson, président de la SRC, en 1977, déclare: « Après un siècle d'existence, notre pays fait face à un double défi: le problème angoissant des deux solitudes et la domination culturelle américaine. C'est dans l'incapacité réciproque des deux principaux groupes linguistiques de se comprendre et de partager leur patrimoine culturel que réside le problème capital de la société canadienne. » SRC, A. W. Johnson, Philosophie et plan d'action de la SRC (juin 1977): 3, 8-9. 
l'Ouest sur la question du français sur les ondes. À son retour, Maher apprend la chose et donne des directives contraires pour que l'émission soit diffusée à l'échelle du pays, précisant qu'il est seul responsable de la programmation nationale. Cet épisode de la radio laisse voir un profond malaise au sein de la commission et permet de jauger à quel point les pressions externes peuvent ébranler la solidarité qui animait la commission à ses tout débuts.

\section{2 - L'ADMINISTRATION QUOTIDIENNE DE LA CCR}

L'une des difficultés majeures à laquelle fait face la commission dans l'exercice de son mandat porte sur le recrutement du personnel qui est assujetti à la loi de la fonction publique. D'une part, cette situation n'offre aucune flexibilité en ce qui a trait aux échelles de traitement et, conséquemment, les commissaires ne peuvent engager le personnel hautement qualifié dont ils ont besoin pour certaines tâches. D'autre part, toute nomination doit, selon la loi, être soumise à l'approbation du ministre. En 1933, le parlement vote une nouvelle loi qui affranchit le personnel supérieur de la commission des restrictions salariales de la fonction publique. Malgré ces efforts louables, la CCR éprouve toujours des difficultés, parfois liées au favoritisme et souvent à des mesures jugées discriminatoires à l'endroit des francophones.

Bien que nous ne puissions établir un lien de causalité entre le pouvoir décisionnel qui ressortit à la radio nationale et le pouvoir administratif de la CCR, nous pouvons toutefois porter un regard scrutateur sur la réalité de tous les jours afin de mieux saisir les problèmes liés aux dimensions linguistique et culturelle françaises qui sous-tendent les activités de la commission. Dans une note adressée à Thomas Maher, le secrétaire de la commission, René-P. Landry, dénonce l'injustice qui lui est faite relativement à une demande de reclassification salariale de sa position; il compare ainsi son salaire à celui d'autres secrétaires de la fonction publique:

La Commission de la Radio, en établissant la position de secrétaire, a exigé que cette position soit remplie à titre bilingue [...]. On aurait cru que les attributions en ce qui concerne le salaire auraient été au moins la moyenne de celles accordées aux secrétaires des différentes commissions. Loin de là - il semble [... que] l'on ait décidé de fixer un salaire à un niveau de beaucoup inférieur à celui accordé aux autres secrétaires. Ceci serait dû au fait que la position est remplie par un Canadien français. En effet le secrétaire de la Commission de la radio est le seul officier 
canadien-français dans le service des commissions gouvernementales. Je considère qu'il y a là une injustice criante [...]. Si l'on a jugé à propos de créer, à la Commission du Service civil, une position d'assistant secrétaire au salaire de $\$ 4920$ par année, il me semble qu'à la Commission de la Radio, où il n'y a qu'un secrétaire, ce dernier devrait être rémunéré avec plus de justice et d'équité [...] le salaire qui m'est attribué n'est guère plus de $\$ 200.00$ par mois, ce qui est ridicule ${ }^{37}$.

Si la démission de Maher a favorisé, comme nous l'avons souligné, un repli du français sur les ondes nationales, il est à craindre, au niveau administratif, que l'effet de sa démission ne remette en cause l'équilibre de la représentation bilingue qui prévalait au sein de la CCR. Dans une lettre adressée à Maher, René-P. Landry écrit encore:

Je t'ai soumis une lettre officielle concernant les prérogatives de ma position et je m'aperçois que l'on est en train de me passer un fling flang [sic].

En regardant les filières, [...] je suis tombé sur une lettre à l'adresse de la Commission du service civil recommandant la création d'une position pour Shaver. Cette position est intitulée «Assistant to the Executive ». Le Colonel Steel dans sa requête mentionne que je ne pourrai remplir [tous] les devoirs qui me sont attribués [...]. Si mes devoirs sont écourtés il n'y aura pas question d'augmentation pour moi [...] je crois que toute l'affaire a été montée par le service civil. Les renseignements que Thivierge nous donnait à toi et à moi il y a quelque temps au Château, n'étaient évidemment pas sans fondement. Tu as sans doute constaté le fait qu'il y a ici deux Canadiens [français] qui occupent des positions d'importance. À chaque fois qu'il a été question d'augmentation, des difficultés quasi insurmontables ont été soulevées. Aussi, j'ai bien constaté, comme toi d'ailleurs, qu'aussitôt qu'il s'agit d'un anglais [sic] les appointements [sic] se font d'une façon excessivement rapide et les salaires sont toujours en excédant [sic] de ce que nous recevons $\mathrm{s}^{38}$.

Le constat de telles irrégularités n'étonne pas: dans son rapport soumis au Premier ministre Bennett, le 25 juillet 1933, Gladstone Murray insistait déjà sur la formation d'un comité de sélection pour l'embauche du personnel, sur la mise en place d'une procédure administrative plus rigoureuse de même que sur la définition et l'application de critères pré-

37. ANC, Fonds René-P. Landry, op. cit., « Mémoire à Monsieur Maher - Re: salaire attribué au secrétaire », II (28 mai 1934).

38. Ibid., « Lettre de Landry à Maher », I (25 juillet 1934?). 
cis permettant de rencontrer les exigences de l'organisme responsable ${ }^{39}$. Quelques semaines avant le dépôt du rapport, Landry recommandait aux commissaires la formation d'un comité ayant pour mandat de définir les responsabilités des employés, notamment celles des chefs de service. Jusqu'à présent, écrit-il, les seules responsabilités qui ont été clairement définies sont celles de secrétaire. Il devient impératif qu'une autorité responsable soit mise en place afin que l'administration de la commission soit plus efficace ${ }^{40}$.

Entre 1933 et 1935, la correspondance de Landry avec ses supérieurs immédiats, à savoir les commissaires en poste, révèle de troublantes anomalies au sein de la CCR. La dénonciation «d'intrigues qui se jouent contre l'élément canadien-français », pour reprendre l'expression de Landry, met en relief des faits équivoques dont la complexité ne permet pas toujours au ministre de la Marine, Alfred Duranleau, de trouver une solution à court terme. L'effet gigogne des problèmes du français qui assaillent, de façon générale, le gouvernement Bennett et, de façon spécifique, le ministère de la Marine (parce que responsable de la radio nationale) place au premier chef des préoccupations du ministre de la Marine les irrégularités commises en fonction de l'appartenance linguistique et culturelle des individus dans la gestion des affaires courantes de la CCR (voir ci-contre l'extrait de la lettre de R.-P. Landry au ministre de la Marine, Alfred Duranleau, 2 mai 1935).

C'est sur une note tout aussi déplorable que prend fin le mandat de Maher lors de sa $73^{\mathrm{e}}$ et dernière réunion à la CCR, le 29 août 1934. Le commentaire de Maher consigné dans le procès-verbal de la réunion rapporte que la commission devrait soumettre sans délai au gouvernement la liste complète des nominations existantes et celle des nouvelles recrues suggérées. Cette liste devrait indiquer le nom des personnes, leur position et leur salaire respectif. Maher reconnaît que la CCR, au cours de sa première année d'activités, a fait du mieux qu'elle pouvait pour combler les postes, compte tenu des circonstances difficiles dans lesquelles elle devait travailler. À la suite de nombreuses irrégularités qui ont été commises par le passé, il estime à présent que la commission devrait corriger cette situation en soumettant une nouvelle liste au Gouverneur en conseil. A priori, ce commentaire rapporté au procès-verbal n'a rien de vexatoire. Pourtant, le secrétaire de la

39. ANC, Appendices, National Radio in Canada, op. cit., 7-8.

40. ANC, Fonds René-P. Landry, op. cit., « Memorendum Concerning the Administration of the Radio Broadcasting Commission », I (15 mars 1934); voir Debates (11 mai 1933): 4861, 4871 . 
Extrait de la lettre de René-P. Landry adressée au ministre de la Marine, le 2 mai 1935

«Tel que vous me l'avez demandé, je vous inclus, sur une liste séparée, la classification de la position de secrétaire à la Commission de la Radio ainsi que le salaire qui lui est attribuable [...].

J'attache à la présente une seconde feuille démontrant les traitements payés aux employés canadiens-français de la radio en comparaison avec ceux qui sont payés aux canadiens-anglais occupant les mêmes fonctions. Ce fait démontre clairement que le gouvernement semble avoir deux mesures dans l'attribution des différentes positions. Je sais que vous faites tout en votre pouvoir pour obtenir justice, et je souhaite de tout cœur que vos efforts obtiendront succès, car je vous avoue que la situation est déplorable $[. .$.$] .$

1. Annonceur. M. Aurèle Séguin $\$ 1600$ par année. Le salaire minimum payé à d'autres positions d'annonceurs au sein de la commission est de $\$ 1800$.

2. Publiciste francophone. M. Léopold Houlé, \$4000 par année. Publiciste anglophone évalué à \$5800 par année.

3. Ingénieur radio. Classe 2. J-A. Ouimet, \$2000 par année tandis qu'une position inférieure d'ingénieur radio, classe 3, a été autorisée à $\$ 2500$.

4. Assistant-opérateur radio. G. Hudon, $\$ 1340$ par année tandis que 9 positions similaires ont été évaluées à $\$ 1800$ par année et 1 à $\$ 1540$.

5. Électricien radio. M. C. Denoncourt, $\$ 1540$ par année tandis qu'une autre position similaire a été évaluée à $\$ 1800$.

6. Directeur de la radiodiffusion, section de l'est. J.-A. Dupont, $\$ 5000$ par année tandis que le salaire payé à l'autre position est de $\$ 5200$.

7. Appartements régis [sic] par la fonction publique: Secrétaire. Lt. Col. René-P. Landry, \$3720 par année tandis que des positions similaires au sein de la fonction publique sont évaluées à \$5540 et $\$ 6000$ respectivement.

Source: ANC, Fonds Landry, vol. II, « Lettre Landry/Duranleau». 
commission, R.-P. Landry, indique que « le Président [l'a informé] que ni lui ni Steel ne signeront ni n'accepteront le procès-verbal si la minute soumise par Monsieur Maher est incluse. Il [Charlesworth] me dit qu'il voit dans l'insistance de Monsieur Maher à vouloir laisser cette minute incorporée dans le procès-verbal, un geste d'inimitié envers lui-même et son collègue $\mathrm{e}^{41}$. »

À présent, nous pouvons mieux saisir les raisons qui ont incité Maher à maintenir son commentaire dans le procès-verbal de la $73^{\mathrm{e}}$ réunion de la commission. La liste complète du personnel en place et à venir, en indiquant le nom, le poste et le salaire respectif de chacun, aurait pu constituer une preuve embarrassante sinon accablante pour les deux commissaires appelés éventuellement à rendre des comptes aux autorités gouvernementales qui étaient déjà préoccupées par la question du français.

Au cours de son bref mandat, la CCR a connu des difficultés internes quelquefois liées à la personnalité de ses membres, souvent aux responsabilités et aux tâches qui lui incombaient, à sa structure même et surtout à l'utilisation du réseau national pour des émissions en français. La commission n'a pu résoudre les nombreux problèmes qui ont entravé son essor. Le comité spécial de 1936 formé par le Premier ministre, W. L. Mackenzie King, recommandera l'abrogation de la loi de 1932, mettant ainsi fin à la CCR. L'application des recommandations de ce comité aboutira, en 1936, à la création de la Société Radio-Canada.

\section{3 - LA CRÉATION DE LA SRC ET LES PREMIERS BALBUTIEMENTS DU RÉSEAU FRANÇAIS}

La loi de 1936 qui assigne à Radio-Canada une mission d'utilité publique ne constitue pas une panacée au problème du français sur les ondes. Il faudra attendre en 1937 pour voir apparaître, au Québec, ce qui constitue le premier noyau du réseau français de Radio-Canada. Dans cette province, le nombre des stations ne représente que le tiers de celles de l'Ontario et les dépenses consacrées au développement de la radio à Montréal sont deux fois et demie inférieures à celles de Toronto. La nouvelle société d'État n'ignore certes pas cette déficience et encore moins l'épineux problème du français sur les ondes auquel elle devra accorder une attention particulière.

41. ANC, Fonds René-P. Landry, op. cit., « Mémoire: procès-verbal de la Commission », II (10 septembre 1934): 4. 
Dès le début de ses activités, la SRC confie respectivement à Montréal et à Toronto la responsabilité de deux grands secteurs opérationnels du réseau national, à savoir le développement technique et la planification de la programmation nationale. Ottawa, qui s'occupe de l'administration générale, demeure le centre de prise de décision. Cette répartition entre Montréal et Toronto renvoie à d'autres considérations importantes que la SRC ne peut certes pas négliger. Montréal, véritable foyer de la culture francophone au pays, prend rapidement conscience de la portée culturelle de la radio. La politique mise de l'avant par la CCR en matière de programmation nationale n'a fait que renforcer son expertise, eu égard à la qualité de la production d'émissions en français, à la reconnaissance de nouveaux talents et à l'accent mis sur la production en direct ${ }^{42}$. Toronto, qui a désormais la responsabilité de la programmation nationale de langue anglaise, devra contrebalancer la présence d'émissions américaines en territoire canadien. On se souviendra que des opposants de la CCR avaient soutenu que la présence du français sur les ondes favorisait l'écoute d'émissions américaines. Cette menace, désormais écartée avec la création d'un réseau français, impose à Toronto un défi de taille: modifier les comportements des habitués de la radio américaine (et ils sont nombreux) tout en favorisant, par le biais de la radio, une culture et un sentiment d'appartenance canadiens. Au dire même de la SRC, « les premières années furent consacrées presque exclusivement à répondre aux exigences technologiques et financières découlant de l'édification d'un réseau; la programmation passa au second rang. Pour répondre à la forte demande d'émissions américaines, il fallut importer les émissions auxquelles les Canadiens s'étaient habitués en écoutant les stations américaines frontalières. Pour favoriser équilibre et excellence, on acheta des émissions parlées et de musique sérieuse produites en Angleterre et aux États-Unis $[\ldots]^{43}$. »

42. Pour une meilleure compréhension de ce propos, voir Alain Canuel, «Les télécommunications à Montréal entre 1846 et 1946 », Scientia Canadensis, 16,1 (1992): 5-24; Albert A. Shea, Broadcasting, the Canadian Way (Montreal, Harvest House, 1963), 45-46; SCRB (1942), 298-299.

43. Société Radio-Canada, Radio-Canada: vue d'ensemble, Mémoire soumis au CRTC à l'appui des demandes de renouvellement des licences d'exploitation des réseaux (mai 1978), 7071; en ce qui a trait aux émissions américaines sur les ondes canadiennes-anglaises, voir les références suivantes: ANC, Fonds Diefenbaker, MG26, 5: 4503 où l'on affirme, en mai 1946, que la SRC retransmet chaque semaine au réseau Trans-Canada 86 émissions américaines en comparaison de 27 d'origine canadienne et au réseau Dominion, 18 émissions américaines en comparaison de 3 émissions d'origine canadienne; Greg Marc Nielsen, Le Canada de Radio-Canada: sociologie critique et dialogisme culturel (Toronto, Éditions du Gref, coll. « Theoria », no 4, 1994), 73; Gilbert Maistre, «L'influence de la radio et de la télévision américaines au Canada », Recherches sociographiques, 12,1 (1971): 51-77, voir particulièrement les pages 56-57. 
Une autre considération liée au développement du réseau français repose sur la puissance émettrice des stations de la SRC. En 1937, la société d'État fait construire deux émetteurs d'une puissance de $50 \mathrm{~kW}$ dont l'un à Toronto (CBL-anglais) et l'autre à Montréal (CBF-français) faisant ainsi passer le rayonnement national de 40 à $76 \%$ de la population. La SRC peut ainsi desservir les deux principaux centres stratégiques de la radiodiffusion, en plus des vastes territoires ruraux situés en périphérie. Le nouveau plan de développement de Radio-Canada accentue toutefois le problème de l'interférence à l'échelle du continent et des changements importants aux niveaux de la fréquence et de la puissance de certaines stations canadiennes devront être apportés ${ }^{44}$.

La mise en service de la station CBF de Montréal constitue le noyau principal du réseau français de Radio-Canada. Cependant, le nombre de stations appartenant ou affiliées à la société d'État ne justifie pas à lui seul l'efficacité du réseau français, car son rendement est aussi fonction de la puissance émettrice et de l'emplacement des stations. En 1933, six stations, affiliées ou louées par la CCR, diffusent sur une partie du territoire québécois les émissions du réseau. En 1939, la situation n'a guère progressé, puisque la faible puissance émettrice des stations ne permet pas de couvrir l'ensemble des régions du Québec (tableau 2). À cet égard, la station CRCS de Chicoutimi s'avère un bon exemple. Comme nous l'avons déjà souligné, la CCR a établi, au début de ses opérations, cinq stations nationales respectivement à Montréal, Toronto, Vancouver, Ottawa et Chicoutimi. La station chicoutimienne fonctionne alors avec une puissance émettrice de 100 watts. En 1937, le journaliste du Progrès du Saguenay, Émile Langis, écrit: «Malheureusement, le rayonnement assuré de ce poste ne dépasse guère 30 milles. Au-delà de cette limite, la réception est faible, souvent embrouillée, parfois absolument nulle. C'est dire que les populations du Saguenay et du nord du Lac St-Jean, isolées du reste de la province, ne sont desservies par aucun poste canadien. Il se trouve que, dans nombre de villages canadiens-français, ce sont les postes américains seuls qui

44. Il est important de noter que, par l'entremise du ministre des Transports, la SRC va entreprendre des démarches pour réunir les gouvernements de 16 pays d'Amérique dont le Canada, les États-Unis, le Mexique et Cuba en vue d'en arriver à une entente définitive sur l'attribution des fréquences et la suppression de l'interférence. Cette initiative aboutira, en novembre 1937, à la conférence de La Havane et permettra à la société d'État de développer un plan de radiodiffusion nationale en conformité aux fréquences qui lui sont attribuées. En vertu de l'accord de La Havane, le Canada obtient six longueurs d'ondes libres en plus d'autres fréquences limitées ou partagées qui lui permettront au fil des ans de mieux s'organiser sur son propre territoire. 
ont droit de citét5. » Il faudra attendre en 1942 pour constater une augmentation significative de la puissance émettrice de la station chicoutimienne, laquelle pourra désormais rejoindre «au-delà de 100000 personnes et [diffuser] ses émissions sur une superficie d'environ 3000 milles carrés à comparer à un champ d'audition de 300 milles carrés [qu'elle] desservait auparavant ${ }^{46} »$.

Fort heureusement, la faiblesse de la puissance émettrice du réseau français au Québec, entre 1932 et 1939, se trouve contrebalancée par la qualité de ses émissions. Malgré une diffusion nationale limitée au cours de cette période, la qualité, l'originalité et l'apport culturel des émissions québécoises auront contribué de façon significative à la survie de la radio française au pays. Thomas Archer, journaliste au Montreal Gazette, a décrit peut-être mieux que quiconque cette réalité:

Bien que [la production française du réseau] emprunte une méthode ou une technique américaine, son expression demeure purement canadienne [...]. Il n'y a pas d'importation. L'influence de la France est négligeable [...]. Les grands systèmes américains furent la convoitise d'ambitieux Canadiens anglais cependant que la porte était fermée au Canada français à cause de la langue et de la tradition de ce peuple. Le Canada français a ainsi développé son propre créneau depuis le début de la radio. Il en récolte aujourd'hui toute la richesse $\mathrm{r}^{47}$.

Les minorités francophones des autres provinces canadiennes qui réclament une juste part du français sur les ondes nationales connaîtront, elles aussi, des déficiences structurelles en plus d'une nette insuffisance d'émissions en français. En mars 1933, le secrétaire-général de l'Association d'éducation des Canadiens français du Manitoba, J.H. Daignault, fait part de son indignation au président de la CCR: « la

45. Émile Langis, «CRCS-Chicoutimi », Progrès du Saguenay, 4 mars 1937.

46. Archives CBJ (Chicoutimi), Document sonore 1.2, «Inauguration des nouveaux studios de CBJ, 1943 ». Allocution prononcée par René Morin, président du Bureau des gouverneurs de Radio-Canada. Il est à noter que l'augmentation de la puissance de CRCS (devenue CBJ en 1939) est due aux changements survenus à la suite de l'accord de La Havane. Sur cette question, voir SCRB (1942), 188-189, 368-369; SCRB (1946), 145, 213-214; SCRB (1947), 59-60; Canada, Ministère des Transports, Accord régional de radiodiffusion de l'Amérique du Nord: signé à la Havane le 13 décembre 1937. Instrument de ratification du Canada déposé à la Havane le 22 décembre 1938 (Ottawa, Imprimeur du Roi, 1939).

47. Thomas Archer, «French Canada Creates Own Broadcasting System », Montreal Gazette, 20 janvier 1939; voir également SCRB (1942), 298; ANC, Fonds Diefenbaker, loc. cit., 5: 4503; Alexander Toogood, Broadcasting in Canada: Aspects of Regulation and Control, 19231969 (Ottawa, Canadian Association of Broadcasters, 1969), 7 et ss.; Shea, Broadcasting, the Canadian Way, op. cit., 45 et ss. 
TABLEAU 2

STATIONS QUÉBÉCOISES AFFILIÉES OU LOUÉES PAR LA CCR EN 1933 ET 1939

\begin{tabular}{llc|llc}
\hline \multicolumn{4}{c}{1933} & \multicolumn{4}{c}{1939} \\
\hline Montréal & $\begin{array}{l}\text { CFCF et } \\
\text { CRCM }\end{array}$ & --- & Montréal & CBF et CHLP $5 \mathrm{~kW} / 5 \mathrm{~kW}$ \\
Chicoutimi & CRCS & 100 watts & Chicoutimi & CBJ & 100 watts \\
Québec & CHRC & 1000 watts & Québec & CBV & 1000 watts \\
Hull & CKCH & 100 watts & Hull & CKCH & 100 watts \\
New Carlisle & CHNC & 100 watts & New Carlisle & CHNC & 1000 watts \\
Noranda & CKRN & 100 watts & Noranda & CKRN & 100 watts \\
& & & Val d'Or & CKVD & 250 watts \\
& & & Rimouski & CJBR & 1000 watts \\
& & & $\begin{array}{l}\text { Ste-Anne- de- CHGB } \\
\text { la-Pocatière }\end{array}$ & 100 watts \\
\hline
\end{tabular}

* En 1936, Radio-Canada s'identifie encore par les lettres CRCM, mais diffuse sur l'antenne CFCF.

Sources: ANC, Fonds Bennett, MG26K, vol. 590: 365267; Archives SRC (Montréal). Note: Les différents documents consultés sur les stations louées ou affiliées révèlent des différences notables. Les sources secondaires suivantes ne sont mentionnées qu'à titre d'information: SCRB (1939), 161-162; Hélène Eck, dir., La guerre des ondes: histoire des radios de langue française pendant la Deuxième Guerre mondiale (Paris/Montréal, A. Colin/Hurtubise HMH, 1985); James Sutherland Thomson, Mémoires du Docteur James Thomson, directeur général et du Docteur Augustin Frigon, directeur général adjoint de la Société Radio-Canada au comité d'enquête nommé par la Chambre des communes sur la radiodiffusion (Ottawa, Juin-Juillet 1943), 27.

Commission Canadienne de la Radiodiffusion, dirait-on, n'existe que sur votre papier à lettre, car nous n'avons encore entendu que le Canadian Radio Commission [...]. La Commission Canadienne de la Radiodiffusion, quand elle s'adresse à tout le pays doit le faire en français; autrement, elle n'est pas canadienne ${ }^{48}$. » Les propos de Daignault mettent en relief un aspect important de la programmation nationale: même

48. Archives de la Saskatchewan, Document R-128, 149(c), «J.-H. Daignault à Hector Charlesworth », (27 mars 1933). 
si certaines émissions portent l'étiquette «française », cela ne garantit pas pour autant qu'elles répondent aux attentes de la population francophone. Et pour cause! La CCR qualifie parfois une émission de « française » parce qu'il s'agit d'un concert français ou simplement parce qu'elle est partiellement diffusée en français. «Ce que nous voulons, ce que nous demandons à la commission, d'écrire Antonio de Margerie, chef du secrétariat de l'Association catholique canadiennefrançaise de la Saskatchewan (ACFC), ce sont des syllabes françaises, et non pas de la musique d'orchestre que nous pouvons entendre d'ailleurs [sic] 24 heures par jour et 7 jours par semaine ${ }^{49}$. » Dans un tel contexte, il devient manifeste que la création de la SRC, en 1936, suscite beaucoup d'espoirs chez les francophones hors Québec, en particulier ceux des provinces du Manitoba, de la Saskatchewan et de l'Alberta.

La deuxième phase du plan d'expansion de la SRC qui se concrétise en 1939 avec l'érection de deux émetteurs régionaux de grande puissance, l'un dans les Prairies (CBK, Watrous, Saskatchewan), l'autre dans les Maritimes (CBA, Sackville, Nouveau-Brunswick) doit permettre au réseau français de diffuser sa programmation nationale d'Edmonton à Moncton. Les auditeurs des Prairies demeurent convaincus que la nouvelle station de Watrous, avec sa puissance de $50 \mathrm{~kW}$, sera en mesure de relayer la quasi-totalité des émissions françaises en provenance de Montréal et d'offrir des émissions à caractère régional pour compléter la programmation nationale. Force est de constater que:

Pour la période du 5 au 12 février 1939, avant l'ouverture du poste CBK, la Société a diffusé 75 heures d'émissions dans la province, dont quatre et trois quarts sont des programmes bilingues. Seulement six et demi pour cent de la programmation s'adresse à la minorité de la langue française.

Un an plus tard, le relevé de la semaine du 3 au 9 février 1940 indique deux heures de programmes bilingues et aucune émission française sur un total hebdomadaire de 116 heures d'émission au réseau national ${ }^{50}$.

Dans les Maritimes, la situation n'est guère plus encourageante. Bien que la majorité de la population francophone soit concentrée au

49. Archives de la Saskatchewan, Document $R-128,23(\mathrm{a})$, « Antonio de Margerie à Hector Charlesworth », (17 avril 1933).

50. Laurier Gareau, Le défi de la radio française en Saskatchewan (Régina, Société historique de la Saskatchewan, 1990), 26; Rossel Vien, Radio française dans l'Ouest (Montréal, Éditions Hurtubise HMH, coll. «Communications », 1977), 33-34. 
Nouveau-Brunswick et que l'émetteur de la station CBA puisse rejoindre les minorités francophones de ces provinces, la programmation du réseau correspond, tout au plus, à quelques heures de français par semaine. La reconnaissance d'une programmation complémentaire qui tienne compte des particularités de chacune des régions s'impose désormais comme une nécessité, afin de répondre aux attentes de la population canadienne. La SRC devra donc décentraliser partiellement sa production en cinq régions: la Colombie-Britannique, les Prairies, l'Ontario, le Québec et les Maritimes.

Au-delà de ce découpage géographique, certaines stations ont déjà fait preuve d'initiatives bien avant que la SRC ne décentralise sa production et n'offre à ses auditeurs une programmation dite « régionale ». À preuve, CRCS (devenue CBJ en 1939) compte parmi les premières stations du réseau national à offrir à ses auditeurs des émissions locales: « mais plus encore que nos autres postes de base à travers le pays, CBJ a gardé son tempérament local, son caractère propre, sa psychologie du milieu, sans oublier son gérant. CBJ, tout en étant Radio-Canada, a été avant tout et surtout Chicoutimi et a mieux contribué ainsi à enrichir d'une note particulière le patrimoine de la radiophonie d'expression française ${ }^{51}$. » N'eussent été de la réalisation d'émissions et de l'augmentation des ressources financières locales pour accroître l'intérêt du milieu et rentabiliser son exploitation, la station CRCS (CBJ) n'aurait pu véritablement s'imposer face aux aléas politiques que connaît la radio à cette époque. Son apport culturel au Saguenay-Lac-Saint-Jean lui aura permis de définir sa propre identité tout en assurant, avec d'autres stations canadiennes, la survie du réseau français ${ }^{52}$.

En dépit des nouveaux émetteurs mis en service en 1939, le réseau français de Radio-Canada n'a pas de véritables maillons à l'extérieur du Québec qui lui permettraient de développer une programmation nationale à partir de différentes régions du pays. Les stations québécoises n'ont pas encore atteint leur pleine maturité qu'elles polarisent déjà nombre d'activités radiophoniques nationales. Pour l'heure, une telle contribution du Québec consolide la position du réseau français, mais elle comporte aussi un effet pervers vis-à-vis d'autres provinces canadiennes: les stations CBK (Prairies) et CBA (Maritimes) constituent

51. Archives CBJ (Chicoutimi), Document sonore 1.14, «25e anniversaire de CBJ et de ses deux fondateurs: Vilmond Fortin, J. E. Roberts », 26 juin 1958. Allocution de Gérard Lamarche, directeur de la SRC pour la province de Québec.

52. Voir Alain Canuel, «La publicité radiophonique au Saguenay et la station CRCS (CBJ) », Saguenayensia, 35,1 (janvier-mars 1993): 16-25. 
une sorte d'appendice du réseau et joueront, pour un certain temps encore, un rôle secondaire quant à l'épanouissement du réseau national français, sans compter qu'elles devront assurer leur propre développement régional. Bref, ces stations sont traitées en parent pauvre lorsque nous les comparons à celles du Québec.

Dans une lettre datée du 12 janvier 1940, Augustin Frigon, directeur-adjoint de la SRC, fait remarquer à l'abbé Maurice Baudoux, ancien président de l'ACFC, que: "Nous avons un bien grand pays et cela présente, à certains moments, des inconvénients très sérieux. Par exemple, le coût de transporter les programmes de la province de Québec à travers le Canada en double, relayés par notre réseau national, serait absolument prohibitif ${ }^{53}$. » L'argument de Frigon sous-entend qu'il y aurait un prix à payer pour rendre équitable le développement d'un double réseau au Canada. Cela suppose également que la SRC doive faire des choix équilibrés, compte tenu de son budget. Mais alors, pourquoi Frigon n'a-t-il pas acquiescé à la proposition de l'abbé Baudoux qui suggérait d'embaucher un commentateur canadien-français à CBK pour réaliser des économies au niveau du réseau et du même coup satisfaire les minorités francophones des Prairies ${ }^{54}$ ? Nous ne saurions débattre de cette question puisqu'elle nécessite une analyse approfondie du développement de la radio au cours de la Deuxième Guerre mondiale. En contrepartie, l'affirmation que nous avons faite en début d'analyse, à savoir que la création d'un réseau français constitue une véritable enclave en territoire national trouve ici toute sa justification.

La Seconde Guerre mondiale marque une nouvelle étape de l'évolution de la radio au Canada avec la création, en 1944, d'un troisième réseau d'État appelé Dominion et destiné à permettre un deuxième choix d'émissions de langue anglaise. Celui-ci compte 35 stations tandis que le réseau initial créé en 1936 totalise 34 stations pour un total de 69 stations de langue anglaise. Le réseau français ne possède que 13 stations, ce qui représente à peine $19 \%$ de l'ensemble des stations, tous réseaux nationaux confondus. Bien que le cadre de notre étude ne nous permette pas d'aborder l'évolution de la radio au cours de la Deuxième Guerre mondiale, nous pouvons tout de même nous interroger sur le

53. Archives de la Saskatchewan, Document R-128, dossier 23(a), «Augustin Frigon à Maurice Baudoux », 12 janvier 1940. La même raison sera évoquée par la commission Massey dix ans plus tard. Voir Vincent Massey, Commission royale d'enquête sur l'avancement des arts, lettres et sciences au Canada (Ottawa, Imprimeur de la Reine, 1951), chapitre 18, article 71, 346.

54. Archives de la Saskatchewan, ibid., «L'abbé Maurice Baudoux à Augustin Frigon », 31 janvier 1940. 
bien-fondé d'un troisième réseau. Compte tenu des coûts élevés que représente la retransmission des «programmes de la province de Québec à travers le Canada, en double », la SRC pouvait-elle vraiment se permettre financièrement de créer, en 1944, un troisième réseau pancanadien?

\section{CONCLUSION}

Les administrateurs de la SRC qui orientent les activités du réseau français de façon à ce qu'elles soient développées là où le besoin est justifié ne font que transposer la vision politique fédérale du moment. En reprenant une politique caractérisée par la duplication, comme celle utilisée dans le cas des billets de banque, la SRC entend trouver un modus vivendi en évitant l'interpénétration des deux langues et des deux cultures que prônait ouvertement Thomas Maher. Rétrospectivement, pouvons-nous estimer réaliste la vision de la SRC et utopique celle de Maher ou vice-versa? Pouvons-nous penser qu'un instrument social et culturel comme la radio aurait permis de rapprocher les deux peuples fondateurs? À ce propos, le président de la CCR, Hector Charlesworth, s'exprime en ces termes:

Au cours de ma carrière de Président, j'aurais souhaité que certains politiciens de langue anglaise fussent aussi tolérants que leurs compatriotes canadiens-français. S'il y a un sentiment séparatiste au Québec, cela est dû en grande partie à une étroitesse d'esprit de certains groupes canadiens-anglais.

En faisant face au problème de l'annonce d'émissions dans les deux langues [les fameuses 30 secondes!] [...] j'ai toujours été convaincu que l'unité nationale aurait fait un grand pas en avant si les deux races avaient favorisé, de façon réciproque, une certaine compréhension de la langue de l'autre. Assurément, le Canada français a fait plus que la moitié du chemin dans son effort de compréhension du Canada anglais ${ }^{55}$.

L'évolution de la radio publique d'expression française entre 1932 et 1939 ne représente qu'un épisode de l'histoire des deux peuples fondateurs qui cohabitent dans un même pays depuis la conquête de la Nouvelle-France. Il ne nous appartient pas ici de jauger les rapports sociohistoriques entre ces deux peuples sur la base de la radio nationale. Les paramètres sur lesquels s'appuie notre analyse suffisent tout au plus à saisir la complexité de l'évolution de la radio nationale à cette époque.

55. Charlesworth, I'm Telling You..., op. cit., 98-99. 
Nonobstant, nous reconnaissons que d'autres facteurs auxquels nous avons fait allusion auraient sans doute élargi notre propos et, du même coup, fourni un contrepoint aux pressions exercées sur la radio d'expression française. Par exemple, certaines actions ponctuelles, telle cette pétition en faveur du français sur les ondes nationales qui témoigne de l'intérêt des populations francophones des Prairies ou encore ces lettres de protestations de personnes influentes ou d'organismes francophones à l'échelle du pays demeurent certes intéressantes d'un point de vue complémentaire, mais non essentielles à notre propos. Il y a aussi les ratés que nous avons passés sous silence et qui n'ont pas permis d'orchestrer une campagne de sensibilisation du français capable de faire échec aux ténors de la radio nationale unilingue (anglaise) ou de développer une stratégie pouvant influencer la pensée politique de l'heure. Il y a enfin les personnes de langue et de culture différentes qui, dans l'exercice de leurs fonctions quotidiennes à la radio, ont fait preuve de convivialité et de tolérance les uns vis-à-vis des autres ${ }^{56}$.

Étant définie par rapport à l'unité nationale et se développant selon des particularités géographique, économique, linguistique et culturelle, la radio publique nationale, entre 1932 et 1939, se situe au carrefour de forces et d'influences multiples. La vision de l'unité nationale qui se dégage de la radio s'appuie, comme nous l'avons déjà précisé, sur diverses interprétations selon les intérêts ou les convictions qu'on y défend. Mais encore faut-il qu'il y ait une volonté réelle, qu'elle soit politique ou autre, pour que la radio nationale puisse se refléter dans le bilinguisme et le biculturalisme canadiens. À ce titre, le discours qu'a prononcé Gladstone Murray, le 22 mars 1937, devant le Cercle canadien de Montréal, alors qu'il occupait le poste de directeur général à la SRC, aurait pu constituer le point d'ancrage d'une véritable radio nationale: «[...] dans ce pays, nous sommes tellement privilégiés de bénéficier de l'histoire de deux écoles de culture que sont les langues anglaise et française que nous devrions tirer profit de cet avantage et, dans cette optique, la $\mathrm{SRC}$ se ferait un devoir public de participer à ce développement ${ }^{57}$. »

56. À titre d'exemple, mentionnons messieurs Vilmond Fortin et James E. Roberts, respectivement directeur et ingénieur en chef de la station CRCS (CBJ) de Chicoutimi qui, pendant près de 35 ans, ont démontré «que le problème de la langue ou de la race ne [les] a pas empêché de s'entendre et de travailler en harmonie ». Archives CBJ (Chicoutimi), Document sonore 1.45, «Soirée d'adieu pour James E. Roberts, 22 mars 1968 à l'hôtel Chicoutimi », citation de Vilmond Fortin; voir également, SCRB (1944), 293.

57. « The Voice of Canada in the Air », Ottawa Journal, 6 avril 1937, 4; « Finite Broadcasting Policy Makes Canada Bilingual », Evening Telegram, 7 avril 1937. 
En concevant la radio canadienne comme un instrument public pouvant tirer profit des deux cultures, des deux littératures et des deux pensées, Murray a posé les jalons qui serviront de réflexion à la Commission royale d'enquête sur la radio et la télévision $(1957)^{58}$.

58. Robert Fowler, Rapport de la Commission royale d'enquête sur la radio et la télévision (Ottawa, Imprimeur de la Reine, 1957), voir particulièrement les pages 34, 272 et 276. 